\title{
Reaction to retractions
}

\author{
Retracting a paper is perhaps the most unpleasant task a journal has to face, particularly if the retraction involves \\ scientific misconduct. With the number of retractions on the rise, an improved mechanism to deal with misconduct \\ is necessary.
}

$\mathbf{O}$ ur September 2003 editorial "The long road to retraction" (Nat. Med. 9, 1093, 2003), written in the aftermath of a particularly problematic case, explained the way in which our journal handles retractions and discussed some of the ramifications that result from removing a paper from the scientific record.

In the editorial we acknowledged that failing to retract a flawed study lends it credibility that it no longer deserves. We also stated that retracting a paper without a proper investigation can unjustly destroy the author's reputation. We indicated that we would retract a paper without the authors' consent only in very extreme circumstances and highlighted the need for all the authors to agree to a retraction before proceeding. And we refuted the notion that we are reluctant to retract papers to avoid embarrassment, emphasizing that we take concerns raised about our papers very seriously. Eight years later, our basic stance on retractions has not changed. But in the midst of a flurry of new retractions and accusations of misconduct, some of which affect our journal (see page 1544), we felt it was time to revisit this topic.

Over the past decade, the number of retractions has markedly increased, particularly those that involve misconduct. At the same time, the widespread availability of software to detect data manipulation has enabled anyone interested in taking a close look to identify fabricated data.

So, many more eyes are watching, and this is making it increasingly hard to get away with data manipulation. For example, sites like Retraction Watch (see page 1539) provide a great service to the community by broadly publicizing and analyzing retractions that might otherwise slip into obscurity. But even though Retraction Watch is a great starting point, scientists, institutions and journals still have a lot of work to do on this front.

Nature Medicine frequently receives e-mails with accusations of misconduct concerning published papers and even manuscripts under consideration. For published papers, the whistleblower often expects us to retract the paper immediately, questioning the need to wait for the outcome of an investigation. But things are never that simple. Although we are committed to setting the record straight, not all misconduct accusations are particularly well supported.

If you are going to accuse someone of wrongdoing, make sure that you have compelling evidence. Simply asking us to withdraw a study because "all the data are made up" is not helpful. And if you have strong evidence, don't alert only the journal, also alert the relevant authorities at the institution of the scientist in question-for example, the dean, the department chair and the office of research integrity. These authorities are the only ones who have the jurisdiction and ability to carry out a formal investigation. Journals simply do not have the resources and capability to do this.

In fact, institutions have a great responsibility when misconduct cases arise, but, disappointingly, not every institution has an office of research integrity. In our experience, it is sometimes difficult to identify the person in charge of investigating problematic studies, particularly in Asia and, to some extent, in Europe. Moreover, some institutions are too quick to dismiss allegations against their staff. Whether this type of denial is on the wane is hard to say, but the steady stream of misconduct cases at even the most respected institutions must result in some tightening of ethical oversight and improved mentoring for young scientists.

Scientific journals, including Nature Medicine, must also improve their handling of accusations of misconduct, as they are in a key position to prevent flawed studies to make it into the scientific record in the first place. It must be said, however, that despite a heightened sensitivity of editors and reviewers to data manipulation, the peer-review process is not designed as a forensic examination of the evidence, and it would be naive to think that this process is foolproof.

Instead, software to detect data manipulation, although not a panacea, needs to be used as proactively as possible. In this regard, Nature Medicine will start using software of this type to check all manuscripts slated for publication. We will also start requiring submission of complete gels at manuscript acceptance, similar to the requirement of our sister journal Nature Cell Biology.

Journals should also explore ways to work together to clamp down on scientists who have a record of submitting manuscripts with questionable or clearly falsified data. Even though journals are bound by the confidential nature of the editorial process, it might be possible to exchange information in a restricted and careful manner about researchers or studies under scrutiny, both before and after publication. Although the creation of a central database with detailed information about the studies in question poses serious logistical challenges, such a database could be an invaluable resource for the fight against misconduct.

It is in their own self interest and for the benefit of the entire scientific enterprise that journals must do all they can to weed out scientific misconduct and mitigate its effects when it occurs. But journals can't do it all; clamping down on misconduct requires a concerted effort from scientists, institutions and journals. We urge the whole community to get behind this effort in a responsible manner. 